\title{
An Interference Identification Algorithm for Electric Energy Data Acquire System
}

\author{
Li Qilin ${ }^{1,}$, , Lu Hua ${ }^{2}$,Qin Jian ${ }^{1}$, Li Fangshuo ${ }^{1}$, Xiang Jingrui ${ }^{1}$ \\ $1,3,4,5$ Metering Center of Sichuan Electric Power Corporation, Chengdu, China \\ ${ }^{2}$ National Key Laboratory of Science and Technology on Communications, Chengdu, China \\ ali_qi_lin@163.com
}

Keywords: Wireless Communication, Electric Energy Data Acquire System, Interference Detection.

\begin{abstract}
Wireless communication network is a key component of Electric Energy Data Acquire System. With the increasingly complicated communication environment, it is essential to detect the Interference introduced by other systems and provide the priori information for the Subsequent interference suppression. Traditional interference detection algorithms generally only can determine whether the interference is present, or it is able to determine the type of interference, but the computational complexity is high. To solve the above problems, this paper presents an interference detection algorithm, which use the spatial correlation and the time domain correlation of interference signals to determine the existence of interference and the type of interference, respectively. This algorithm has the characteristics of low computational complexity, and the simulation results show that the algorithm can achieve good performance of interference detection.
\end{abstract}

\section{Introduction}

In the complicated wireless environment, with more and more interference, the interference will seriously impacts the performance of the system. In order to facilitate the follow-up signal demodulation process, the receiver is necessary to detect the interference. In this paper, the LTE (Long Term Evolution) system is considered as the wireless public network in the electric energy data acquire system.

The existing interference detection technology can be divided into time-domain detection and transform-domain detection. The time-domain technologies, including the time-domain energy detection [1], the detection based on covariance matrix [2], the detection based on the feature value decomposition of matrix [3], the maximum-likelihood detection [4], the kurtosis detection [5] etc. The time-domain detection algorithm usually use the difference between the interference and noise, including energy, relevance etc. However, the time-domain detection are algorithms which generally only can determine whether the interference is present.

In the transform-domain detection, the frequency-domain detection is researched mostly. For example, the mean value detection algorithm [6], consecutive mean excision (CME) [7]. In [8] and [9], forward consecutive mean excision (FCME) and double threshold interference detection are proposed on the basis of the CME algorithm. The frequency-domain detection uses the frequency characteristic of the interference, the algorithms can not only detect the existence of the interference, some are also able to detect the type of the interference. However, most of the frequency-domain detection algorithm need for iterations. Convergence is a time-consuming process, and computing complexity is also relatively high.

As the LTE base-station has many antennas, this paper proposes an algorithm to detect the existence of interference in spatial-domain and to determine the type of the interference using the time-domain relevance of the interference. The algorithm firstly eliminate the influence of the expected signal at the receiving end, and then take full advantage of the difference between the interference and noise to detect the interference. In additional, the computing complexity of the algorithm is very low. Simulation results are presented to verify the validity of the method, and the 
cumulative probability density distribution (CDF) curves of the judgment with different channel model and different signal-to-noise (SNR) are also illustrated.

\section{System Model}

Electric Energy Data Acquire System consists of three parts: Master station, communication network and collectors, its structure is shown in Fig. 1.Master station is the center of Electric Energy Data Acquire System, which is responsible for controlling the data acquire terminals, processing and sharing information of electric energy, and offering the data interface to services of electric power department and the marketing applications. Communication network provides a communication channel to connect data acquire terminals and master station, including fiber optic private network, $230 \mathrm{MHz}$ wireless private network, CDMA/3G wireless public network, etc.

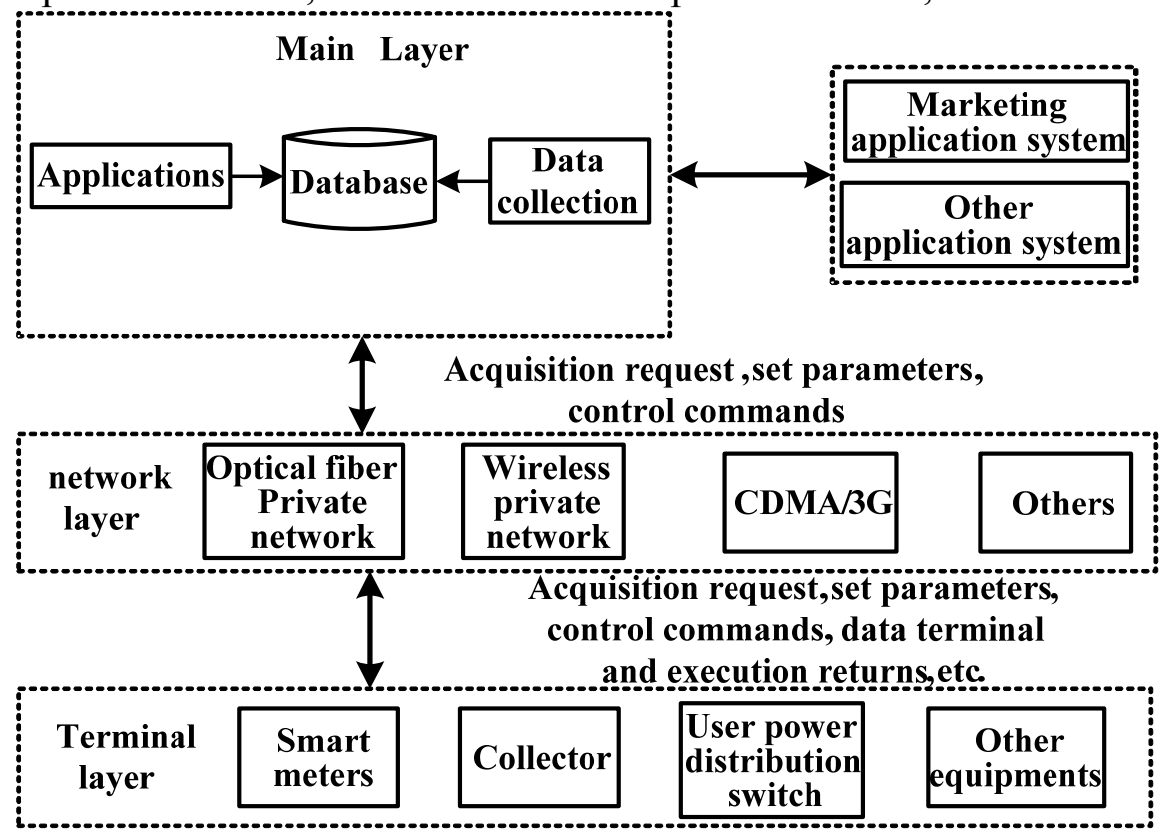

Figure 1 Structure of electric energy data acquire system

The electric energy data acquire system with LTE communication system is considered in this paper. At the base station, Assuming that the number of receiving antennas is $N_{r}$, the number of FFT points is $N$, the total number of carriers that the user can use is $N_{s}$. In order to facilitate description, it is assumed that all the carriers are occupied by the user. After analog-to-digital conversion, removing the cyclic prefix (CP), N-point FFT, subcarrier de-mapping, the frequency domain received vector $Y_{n}$ can be written as

$$
Y_{n}=X h_{n}+u_{n}
$$

Where $X$ is the diagonal matrix, representing the frequency domain launched symbol, $h_{n}$ is channel gain vector, $u_{n}$ is interference plus noise vector, and

$$
u_{n}=j_{n}+n_{n}
$$

Where $j_{n}$ is the interference vector, $n_{n}$ represents the Gaussian White Noise vector, then after $\mathrm{N}$-point IFFT, the time domain interference vector $v_{n}$ can be obtained.

$$
v_{n}=i_{n}+w_{n}
$$


Where $i_{n}$ and $w_{n}$ represent the time domain interference vector and noise vector respectively.

In the text, it is assumed that is DMRS symbol in Eq. $1, X$ is known and $h_{n}$ can be acquired by channel estimation. Therefore, the interference vector $v_{n}$ can be obtained at the receiving end. The algorithm uses $v_{n}$ to detect the existence and the type of the interference for the reason that it can reduce the impact of the expecting signal.

\section{Interference Detection}

Taking into account the need and the computing complexity of the interference detection, an algorithm using the spatial relevance of interference to determine whether the interference exists and the frequency relevance of interference to determine the type of interference is proposed in this paper.

Interference presence detection. Assuming the number of receiving antennas is $M$, we can receive the $M \times M$ spatial relevance matrix $R$ of the received signal, which can be expressed as

$$
R=R_{v}+N_{0} I
$$

Where $R_{v}$ represents spatial relevance matrix of interference, $N_{0} I$ represents spatial relevance matrix of noise. It can be seen that if the interference does not exist, the matrix $R$ is a diagonal matrix. In accordance with the characteristic, it is possible to detect the existence of the interference.

Taking vector $v_{1}, v_{2}, \ldots v_{M}$, and then combining into a $N_{s} \times M$ matrix $V=\left[v_{1}, v_{2}, \ldots v_{M}\right]$. We can obtain an estimated value $R_{s}$ of $R$ from matrix $V$.

$$
R_{s}=\frac{1}{N_{s}} V^{T} V^{*}
$$

Where $V^{T}$ and $V^{*}$ represent the built-in matrix and the conjugate matrix respectively.

The $i^{\text {th }}$ row and $j^{\text {th }}$ column of matrix $R_{s}$ is denoted as $r_{i j}$. We define the parameter $\alpha$ as

$$
\alpha=\frac{\sum_{i=1}^{M} \sum_{j=1, j \neq i}^{M}\left|r_{i j}\right|}{(M-1) \sum_{i=1}^{M}\left|r_{i j}\right|}
$$

Then a threshold $T_{1}$ is been selected, if $\alpha<T_{1}$, interference is not existing, otherwise, interference is existing and interference suppression measures are needed.

Type of interference detection. When interference is presented in the system, there is a need to judge the type of the interference. In this paper, it uses the time domain relevance to determine the type of the interference.

Considering the noise vector $v_{n}$, the $i^{\text {th }}$ element is denoted as $v_{n}[i]$. And the related value is defined as

$$
C[k]=E\left\{v_{n}[i] v_{n}^{*}[i-k]\right\}
$$

In order to estimate $C[k]$, a time domain average is conducted

$$
C_{e}[k]=\frac{1}{N_{s}-1} \sum_{i=k-1}^{N_{s}} v_{n}[i] v_{n}^{*}[i-k]
$$


The parameter $\beta$ is defined as:

$$
\beta=\frac{\sum_{k_{1}}^{k_{2}}\left|C_{e}[k]\right|}{C_{e}[0]}
$$

Then a threshold $T_{2}$ is been selected, if $\beta<T_{2}$, interference is broadband signal, otherwise, interference is narrowband signal.

\section{Numerical and simulation results}

In order to verify the method of interference detection, this section will provides computer simulation results under different conditions.

In the simulation, the number of transmitting and receiving antenna are 1 and 2 respectively, the transmitting bandwidth is $10 \mathrm{M}$, the points of FFT is 1024 , the maximum Doppler shift is $50 \mathrm{~Hz}$, the modulation mode is 16 QAM, the bandwidth of narrowband interference is $400 \mathrm{KHz}$ and the channel estimation is ideal channel estimation.

The CDF performances of interference existence detection with different SNR under different channel are illustrated in Fig. 2. When the SNR is $-10 \mathrm{~dB}$ and the channel mode is EPA, the threshold is set as 0.05 . When the interference is free, the algorithm works incorrectly with the probability of 2 percent, and when the interference is narrowband, the probability to detect the existence is 96 percent, and when the interference is broadband, the probability to detect the existence is 90 percent. When the channel mode is EVA, when the interference is free, the algorithm works incorrectly with the probability of 3 percent, and when the interference is narrowband, the probability to detect the existence is 94 percent, and when the interference is broadband, the probability to detect the existence is 80 percent. When the SNR is $10 \mathrm{~dB}$, whether the channel mode is EPA or EVA, the probability to detect the interference is close to 100 percent.

The CDF performances of interference type detection with different SNR under different channel are illustrated in Fig. 3. When the SNR is same, the value $\beta$ of narrowband interference and broadband interference varies obviously. When the SNR is $-10 \mathrm{~dB}$, the threshold is set as 0.05 , the probability to identify narrowband interference to broadband interference is 10 percent, when the SNR is $10 \mathrm{~dB}$, whether the channel mode is EPA or EVA, the probability to detect the interference is close to 100 percent. 

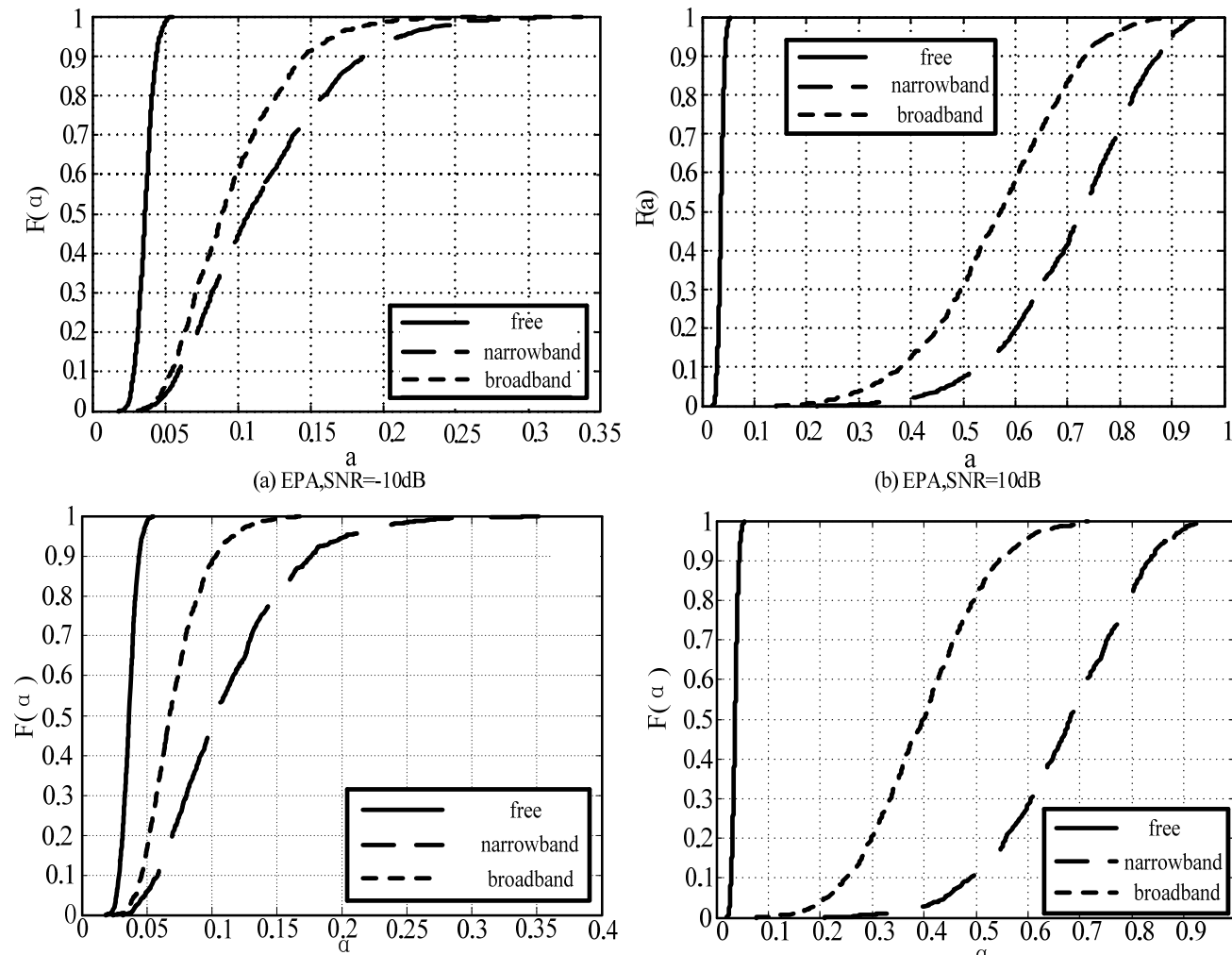

(c) $\mathrm{EVA}, \mathrm{SNR}=10 \mathrm{~dB}$

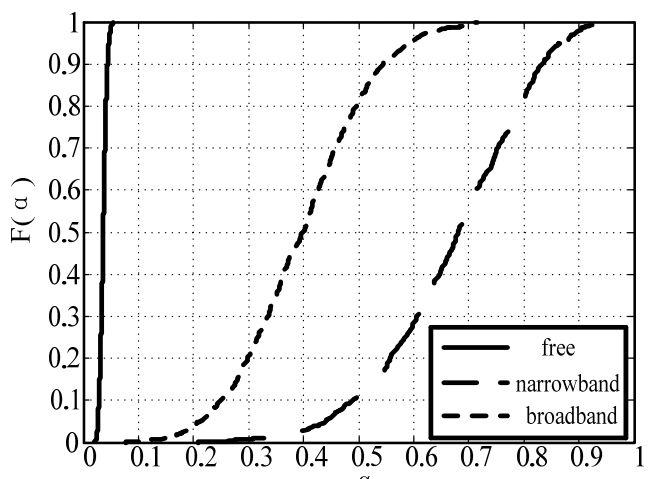

(d) $\mathrm{EVA}, \stackrel{a}{\mathrm{SNR}}=10 \mathrm{~dB}$

Figure 2 CDF performances of interference existence detection with different SNR under different channel
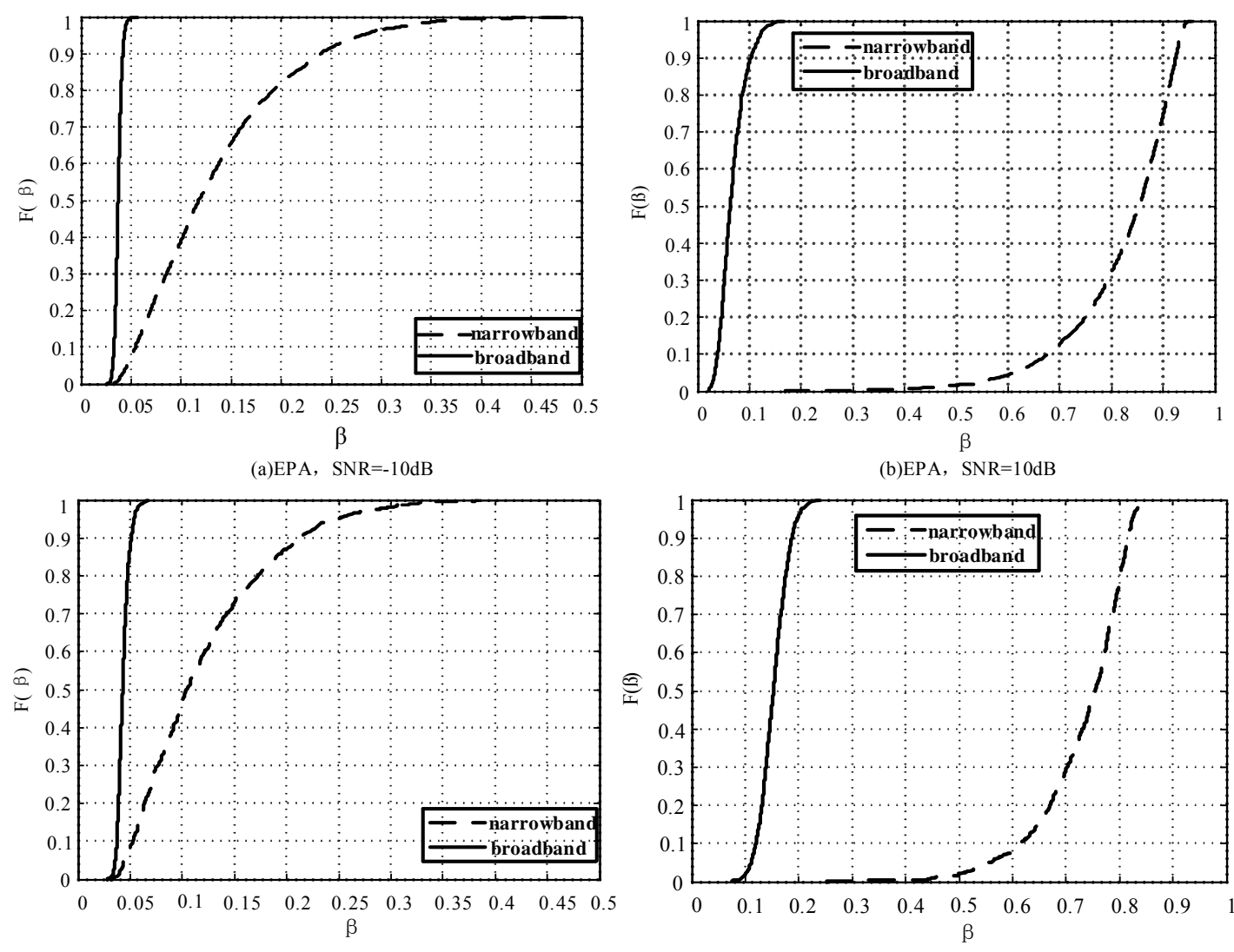

(c)EVA, SNR=-10dB

(d)EVA, SNR $=10 \mathrm{~dB}$

Figure 3 CDF performances of interference type detection with different SNR under different channel 


\section{Summary}

An algorithm is proposed in this paper to detect interference in the electric energy data acquire system which uses LTE system as the wireless public network in this paper, including interference existence and interference type detection. The existence detection uses the spatial relevance difference between interference and noise. While the type detection uses the time domain relevance difference between narrowband interference and broadband interference. Finally, the computer simulations are illustrated to verify the performance of the proposed algorithm.

\section{Acknowledgement}

This work was supported by the Technology Project (No: SGSCDKJLZJKJ1400099) of State Grid Corporation of China.

\section{References}

[1] Qi Ling, Tongtong Li, Modeling And Detection Of Hostile Jamming In Spread Spectrum Systems, IEEE Transactions On Communications vol 57, 6 No June, 2000.

[2] Y . H . Zeng, Y . C . Liang, Eigenvalue-Based Spectrum Sensing Algorithms for Congnitive Radio, IEEE Transactions On Communications vol 57, 6 No June, 2009.

[3] Y . H . Zeng, Y . C . Liang, Spectrum-sensing algorithms for congnitive radio based on statistical covariances, IEEE Transactions OnVehicular Technology, Vol 58, 4 No May, 2009.

[4] F . S . Josep, X . D . Wang, GLRT -based spectrum sensing for congnitive radio with prior information, IEEE Transactions On Communications vol 58, 7 No July, 2010.

[5] Sidharth Misra, Priscilla N . Mohammed, Microwave Radiometer Radio - Frequency Interfence Detection Algorithms: A Comparative Study, IEEE Transactions On Geoscience And Remote Sensing, Vol 47, 11 No November, 2009.

[6] Petti Henttu, A New Interference Suppression Algorithm Against Broadband Constant Envelope Interference, IEEE Transactions On Communications vol 51, 4 No June, 2007.

[7] Petti henttu, Sami Aromaa, Consecutive mean excision algorithm, IEEE 7' H Int . Syinp. On Spread - Spectrum Tcch . \&Appl ., Prague. Czech Republic Sepr . . 2-5, 2002.

[8] Ming Fan, Zeding Wei, Interference Detection In DSSS Systems,Modern Electronic Technology vol 13,2007.

[9] J . Vartiainen, J . J . Lehtomaki, H . Saarnisaari, Double threshold. Based Narrowband Signal Extraction, Vehicular Technology Conference [C], 2005 IEEE 61st Volume 2, page(s): 1288 - 1292. 